\title{
Les mycotoxines dans les produits laitiers
}

\author{
par \\ Claude MOREAU
}

Centre National de la Recherche Scientifique

Laboratcire de Biologie Végétale et de Microbiologie

Université de Bretagne Occidentale - 29279 Brest cedex

\section{Introduction}

\section{L'aflatoxine M}

- Les aflatoxines.

- Sensibilité des bovins aux aflatoxines.

- Métabolisme des aflatoxines.

- Vitesse de métabolisation et quantité d'aflatoxine $\mathrm{M}_{1}$ produite.

- Conséquences pour les dérivés du lait.

- Toxicité de l'aflatoxine $\mathbf{M}_{1}$.

- Conséquences pratiques.

- Législation.

\section{Formation éventuelle de mycotoxines dans les produits laitiers}

- Poudres de lait.

- Lait concentré.

- Fromages.

- Beurre.

Conclusions

\section{INTRODUCTION}

Les mycotoxines sont des substances toxiques pour l'homme et les animaux, élaborées par des moisissures développées dans les denrées alimentaires (Moreau, 1974). Les intoxications liées à ces substances peuvent se manifester par des syndromes fort variés : gastro-entérites, hémorragies, convulsions, paralysies, effets œstrogènes, etc. mais surtout lésions des reins et du foie ; dans certains cas, ces dernières peuvent se traduire par des cancers.

On comprend donc aisément pourquoi la question des mycotoxines préoccupe les hygiénistes et les toxicologues.

Dans le cas des produits laitiers, il convient de distinguer deux catégories de pollution par mycotoxines : 
- d'une part la présence de mycotoxines dans le lait d'animaux ayant consommé des aliments renfermant déjà des mycotoxines ; tel est le cas de l'aflatoxine $M$, particulièrement dangereuse ;

- d'autre part l'élaboration de mycotoxines par des moisissures développées dans le lait sec ou sur des dérivés du lait ; en outre, l'éventualité de la production de mycotoxines par des moisissures utiles à la préparation de certains fromages ne doit pas être a priori rejetée.

\section{L'AFLATOXINE M}

\section{Les aflatoxines}

Les aflatoxines sont des dérivés de la coumarine produits par diverses moisissures, surtout l'Aspergillus flavus Link, un champignon fréquent dans le sol, sur des matières organiques variées, sur les grains et particulièrement sur les graines oléagineuses.

On connaît maintenant plus d'une dizaine d'aflatoxines de formules chimiques voisines. La plus importante et la plus dangereuse est l'aflatoxine $\mathrm{B}_{1}$.

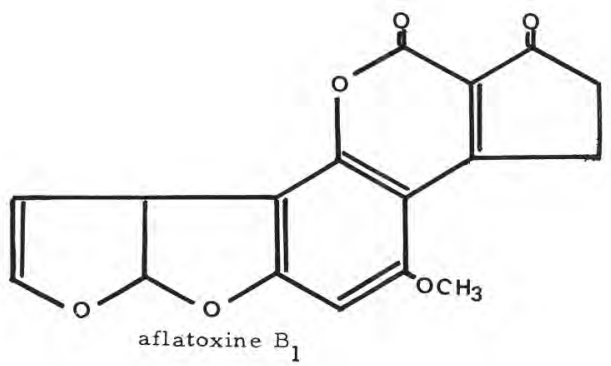

Les amandes d'arachide contiennent souvent des aflatoxines. Lors de la préparation de l'huile, les aflatoxines passent dans les tourteaux, l'huile elle-même en est exempte. Or, ces tourteaux sont une des bases de l'alimentation animale.

\section{Sensibilité des bovins aux aflatoxines}

Tous les animaux ne sont pas également sensibles aux aflatoxines. Les ovins, par exemple, sont remarquablement résistants, éliminant rapidement ces toxines par les urines et les féces.

Chez des vaches laitières de 2 ans, un apport de 0,22 de 0,44 ou de $0,66 \mathrm{mg} / \mathrm{kg}$ d'aflatoxine $B_{1}$ dans la ration quotidienne, n'a que peu d'effets même après 20 semaines de ce régime, si ce n'est de légères lésions hépatiques pour la teneur la plus élevée (Clegg et Bryson, 
1962) ; des rations contenant $2,4 \mathrm{mg} / \mathrm{kg}$ d'aflatoxine $\mathrm{B}_{1}$ ont été données à des vaches âgées de 8 à 10 ans : les effets ne se sont fait sentir qu'après 7 mois de ce régime ; si l'on donne des aliments contenant $2,7 \mathrm{mg} / \mathrm{kg}$ d'aflatoxine $\mathrm{B}_{1}$ à des vaches, on constate seulement une diminution de la lactation entre 20 et $53 \mathrm{j}$ (Allcroft et Lewis, 1963).

Les individus jeunes sont toujours plus sensibles que les adultes aux effets des aflatoxines. Si, à des veaux, on introduit dans la ration 5 à 8 p. 100 de nourriture réputée toxique (ce qui correspond à 0,22 à $0,44 \mathrm{mg} / \mathrm{kg}$ d'aflatoxine $B_{1}$ ) chaque jour pendant 16 semaines, on n'observe aucun signe clinique si ce n'est une légère diminution de poids au cours des 3 premiers mois ; si la nourriture toxique entre pour 18 p. 100 dans la ration quotidienne pendant 16 semaines, cet abaissement du poids est cependant plus sensible et, si l'on prolonge l'expérience, la mort peut s'ensuivre (Allcroft et Lewis, 1963).

Il ne semble pas que l'aflatoxine ingérée par des vaches en gestation ait une répercussion sur la santé du veau : dans les observations expérimentales que l'on possède à ce sujet, les veaux sont nés en parfaite santé.

\section{Métabolisation des aflatoxines}

Dès 1962, on s'est aperçu d'une migration des substances toxiques dans le lait (Allcroft et Carnaghan, 1962, 1963) : si l'on donne à des vaches laitières une alimentation en tourteaux riches en aflatoxines, leur lait est toxique pour le caneton de $1 \mathrm{j}$, animal particulièrement sensible aux aflatoxines.

L'analyse du lait a révélé que s'il renfermait des traces d'aflatoxine $B_{1}$, il contenait surtout un métabolite auquel le nom de "milk toxin " ou aflatoxine M a été donné (De Iongh et al., 1964, 1965).

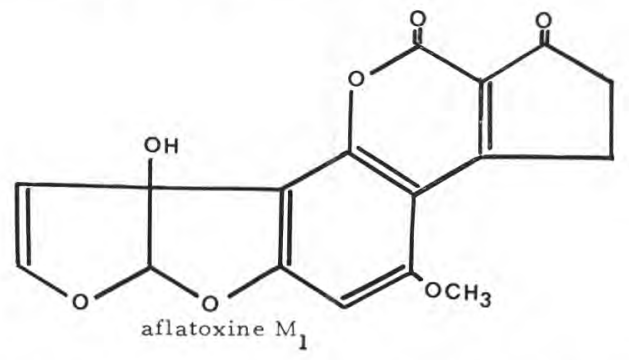

On s'est ensuite aperçu qu'il s'agissait de deux dérivés hydroxylés des aflatoxines $B_{1}$ et $B_{2}$ que l'on a respectivement nommés aflatoxine $\mathbf{M}_{1}$ et aflatoxine $\mathbf{M}_{2}$ (Büchi et Weinreb, 1969 : Holzapfel, Steyn et Purchase, 1966 ; Masri et al., 1967), l'aflatoxine $M_{1}$ étant la plus importante. 
Analysant 165 échantillons de lait, Kiermeier et al. (1975) ont constaté qu'environ 14 p. 100 avaient une teneur en aflatoxine $M_{1}$ supérieure à $0,05 \mu \mathrm{g} / \mathrm{l}$.

Pour être sûr que, dans le lait, il s'agissait de métabolites de l'aflatoxine ingérée, on a administré, par voie orale, de l'aflatoxine B1 à des rates allaitantes ; on a retrouvé dans leur lait de l'aflatoxine $M_{1}$ (De Iongh et al., 1964, 1965).

On a pu retrouver cette aflatoxine $\mathbf{M}_{1}$ dans le lait de chèvre (Delage et Fehr, 1967), dans la bile des rats (Bassir et Osiyemi, 1967), dans les féces et les urines de vaches et de mouton (Allcroft, Roberts et Lloyd, 1968 ; Allcroft, Rogers et al., 1966 ; Jones et Lee, 1958 ; Masri, Garcia et Page, 1969 ; Nabney et al., 1966 ; Nabney et Nesbitt, 1964), dans le sang, l'urine, la bile et les reins de lapereaux (Fehr, Delage et Richir, 1970), dans l'urine des oies et cobayes (Patterson et Allcroft, 1970).

Plus récemment, on a identifié l'aflatoxine $\mathrm{M}_{1}$ dans l'urine d'humains consommant du beurre d'arachide contaminé et dans le lait d'une indienne dans les mêmes conditions (Campbell et al., 1970), ainsi que dans du lait vendu sur un marché sud-africain (Purchase et Vorster, 1968).

Signalons que certaines souches d'Aspergillus flavus sont aptes à produire directement de faibles quantités d'aflatoxine $\mathbf{M}$ sans qu'il y ait eu métabolisation par un organisme (Pai, Bai et Venkitasubramanian, 1975).

\section{Vitesse de métabolisation et quantité d'aflatoxine $\mathbf{M}_{1}$ produite}

La métabolisation des aflatoxines ingérées est assez rapide (Chou Ming-Wu et Tung Ta-Chang, 1969).

Plusieurs essais ont été réalisés.

Si l'on fait absorber à des vaches une seule dose $(0,5 \mathrm{mg} / \mathrm{kg})$ d'un mélange d'aflatoxines ( $B_{1}: 44$ p. $100 ; G_{1}: 44$ p. $100 ; B_{2}: 2$ p. 100) et qu'on analyse régulièrement le lait, l'urine et les féces, on constate que 85 p. 100 de l'aflatoxine détectée dans le lait et l'urine sont excrétés dans les premières $48 \mathrm{~h}$; aucune trace n'est plus détectable dans le lait après $4 \mathrm{j}$. La quantité d'aflatoxine $\mathrm{M}_{1}$ qu'on trouve dans le lait représenterait 0,35 p. 100 de la quantité d'aflatoxine $B_{1}$ ingérée (Allcroft et Roberts, 1968 ; Allcroft, Roberts et Lloyd, 1968 ; Roberts et Allcroft, 1968).

Dans d'autres essais et peut-être aussi en raison de la sensibilité des méthodes d'analyses, des pourcentages légèrement différents ont été obtenus : Lafont (1975) considère que l'élimination d'aflatoxine $M_{1}$ par la mamelle peut atteindre 0,85 p. 100 de l'aflatoxine ingérée ; 
pour Neumann-Kleinpaul et Terplan (1972), c'est environ 1,39 p. 100 de l'aflatoxine $B_{1}$ ingérée qui est excrétée dans le lait sous forme d'aflatoxine $\mathrm{M}_{1}$; selon Kiermeier (1972), c'est 1,5 p. 100 qui est présent.

Pour leur part, Jacquet et al. (1971) ont trouvé seulement quelques microgrammes par litre d'aflatoxine dans le lait de vaches qui consommaient $0,3 \mathrm{mg} / \mathrm{kg}$ d'aflatoxine et 5 à $20 \mu \mathrm{g} / 1$ dans le lait de celles qui absorbaient $1,3 \mathrm{mg} / \mathrm{kg}$ d'aflatoxine.

Le problème se complique si l'on fait consommer aux vaches de l'aflatoxine $B_{1}$ pendant plusieurs jours, ce qui d'ailleurs est plus proche de la réalité dans les élevages industriels. Lors d'une expérience, 4 vaches laitières ont absorbé respectivement $10,50,250$ et $1250 \mu \mathrm{g} / \mathrm{kg}$ d'aflatoxine $\mathrm{B}_{1}$ pendant 2 semaines ; on ne détecte pas d'aflatoxine $\mathrm{M}_{1}$ dans le lait de la vache consommant $10 \mu \mathrm{g} / \mathrm{kg}$ d'aflatoxine $\mathrm{B}_{1}$; on en trouve seulement des traces chez celle qui en absorbe $50 \mu \mathrm{g} / \mathrm{kg}$. Chez celles qui en reçoivent 250 et $1250 \mu \mathrm{g} / \mathrm{kg}$, la quantité d'aflatoxine $\mathrm{M}_{1}$ retrouvée dans le lait s'accroît au cours des 4 premiers jours puis demeure à peu près stable. La production d'aflatoxine $\mathrm{M}_{1}$ cesse $2 \mathrm{j}$ après la fin de l'expérience (Polan, Hayes et Campbell, 1974).

La brebis se comporte un peu différemment de la vache : l'excrétion d'aflatoxıne $\mathbf{M}_{1}$ par les urines et les féces est plus précoce et en moins grande quantité ; par contre beaucoup plus d'aflatoxine $\mathbf{B}_{1}$ est excrétée (Allcroft et al., 1966 ; Lewis, Markson et Allcroft, 1967 ; Nabney et al., 1966).

\section{Conséquences pour les dérivés du lait}

On a évidemment recherché si l'aflatoxine $\mathbf{M}_{i}$ se retrouvait dans le lait sec. Il semble que lors du séchage 75 p. 100 de la toxine soit détruite (Kiermeier, 1973).

Sur 166 échantillons de lait sec variés provenant de 24 producteurs différents, on a constaté par l'analyse, selon les méthodes $\mathrm{AOAC}$, que 8 présentaient de l'aflatoxine $\mathrm{M}_{1}$; mais les quantités détectées $(0,67$ à $2 \mu \mathrm{g} / \mathrm{kg}$ de lait $\mathrm{sec})$ sont considérées par NeumannKleinpaul et Terplan (1972) comme sans danger pour les enfants qui consomment ce lait.

L'analyse d'aflatoxine $\mathrm{M}_{1}$ dans le lait sec a donné lieu à la recherche de méthodes de plus en plus sensibles (Stubblefield et Shannon, 1974) ; on peut actuellement détecter des quantités de l'ordre de $0,2 \mu \mathrm{g} / \mathrm{kg}$ (Jung et Hanssen, 1974).

Lors de la préparation de fromages à partir de lait renfermant de l'aflatoxine $\mathrm{M}_{1}$ on a constaté que 71 à $74 \mathrm{p}$. 100 de la toxine passait dans le babeurre ; par contre, dans le beurre, on n'en retrouve que 16 p. 100 (Stubblefield et Shannon, 1974). 
Dans des recherches systématiques portant sur 222 fromages, Kiermeier (1970) a retrouvé des aflatoxines dans 7 p. 100 d'entre eux ; dans une autre observation, le même auteur n'a trouvé de traces d'aflatoxine que dans 2 fromages sur 115 échantillons prélevés dans le commerce (Kiermeier, 1975).

\section{Toxicité de l'aflatoxine $\mathrm{M}_{1}$}

Pour le caneton de $1 \mathrm{j}$, la $\mathrm{DL}_{\approx}$ par voie orale est de $0,32 \mathrm{mg} / \mathrm{kg}$ pour l'aflatoxine $\mathrm{M}_{1}$ (contre $0,24 \mathrm{mg} / \mathrm{kg}$ pour l'aflatoxine $\mathrm{B}_{1}$ dans les mêmes conditions) (Purchase, 1967). Ainsi les toxicités aiguës de ces deux aflatoxines diffèrent-elles peu.

Les lésions provoquées sur le foie du caneton par l'aflatoxine $\mathbf{M}_{1}$ sont semblables à celles que cause l'aflatoxine $\mathrm{B}_{1}$; de faibles doses de $M_{1}$ peuvent déclencher une dégénérescence progressive des cellules du foie et une prolifération des canaux biliaires (Purchase, 1967). Enfin, on considère que, pour la truite par exemple, l'aflatoxine $\mathbf{M}_{1}$ est incontestablement un carcinogène potentiel du foie au même titre que B. (Sinnhuber et al., 1970).

L'aflatoxine $\mathrm{M}_{2}$ est moins toxique que $\mathrm{M}_{1}$; la DLø pour le caneton de $1 \mathrm{j}$ est 1,248 $\mathrm{mg} / \mathrm{kg}$ (Purchase, 1967).

\section{Conséquences pratiques}

Selon les auteurs, c'est donc 0,35 à 1,5 p. 100 de l'aflatoxine ingérée par des vaches que l'on retrouve dans le lait sous forme d'aflatoxine $M_{1}$ aussi dangereuse que l'aflatoxine $B_{1}$.

On peut être d'autant plus inquiet qu'une partie de ce lait est utilisée en nourriture infantile et que les enfants sont beaucoup plus sensibles que les adultes aux effets des aflatoxines.

Remarquons cependant avec Abrams qu'un enfant de $5 \mathrm{~kg}$ devrait absorber 271 de lait par jour, s'il s'agit de lait de vaches consommant par jour $2 \mathrm{~kg}$ de tourteaux d'arachide contenant $4 \mathrm{mg} / \mathrm{kg}$ d'aflatoxine $\mathrm{B}_{1}$ pour présenter les mêmes symptômes que les canetons, en supposant que la sensibilité de l'être humain et celle des canetons soient identiques.

Il convient de ne pas s'alarmer outre mesure. Il est bien prouvé que les quantités d'aflatoxines ingérées par les vaches sont très variables d'un élévage à l'autre, ces aflatoxines n'étant d'ailleurs pas apportées uniquement par les tourteaux d'arachide. Or, dans notre économie actuelle, dans les coopératives, le lait d'une ferme est généralement mélangé au lait provenant d'autres fermes de sorte que dans le lait pasteurisé du commerce les teneurs en aflatoxines sont normalement si faibles qu'on ne peut les détecter. 


\section{Législation}

Le problème de la présence de substances dangereuses dans le lait comme les aflatoxines $\mathrm{M}$ a retenu l'attention des pouvoirs publics et le Conseil Supérieur d'Hygiène s'est récemment penché sur la question (séance du 28 oct. 1975).

L'arrêté du 2 février 1973 (paru au Journal Officiel du 15 février 1973) réglemente la teneur en aflatoxines tolérables dans les aliments destinés aux animaux. Un texte est en préparation pour l'alimentation humaine. Mais il conviendra de mettre au point une méthode d'analyse fiable susceptible d'être utilisée par les professionnels.

A l'échelle internationale divers règlements sont en cours d'élaboration, notamment en Allemagne ; mais on se heurte à de nombreuses difficultés d'application (échantillonnage, méthodes d'analyses, spécificité, taux...).

\section{FORMATION EVENTUELLE DE MYCOTOXINES DANS LES PRODUITS LAITIERS}

Des accidents de mycotoxicoses sont susceptibles de se produire par consommation de produits laitiers. On rencontre en effet chez ceux-ci de nombreuses moisissures aptes à élaborer des mycotoxines. Bien qu'à notre connaissance aucun accident ne puisse leur être imputé avec certitude, il convient de ne pas nier le danger et être prudent en cette matière.

\section{Poudres de lait}

Ce sont surtout les poudres lactées qui, malgré leur faible teneur en eau ( 3 à 4 p. 100 normalement), recèlent des moisissures lorsqu'elles sont préparées ou stockées dans de mauvaises conditions (Cooke et Brazis, 1968).

Le tableau 1 fournit les résultats de l'analyse mycologique de 30 échantillons originaires de Normandie, Bretagne et Vendée parvenus à notre laboratoire au cours des récentes années (1).

(1) Cette étude, réalisée avec la collaboration technique de Marie-Yvonne Jan et Marie-Anne Le Bras, entre dans le cadre de la convention de recherches 73-49 du Ministère de la Qualité de la Vie : cheminement des polluants dans les chaînes alimentaires. 


\section{TABLEAU 1}

Pollution fongique de poudres de lait.

Résultat global de l'analyse de 30 échantillons

\begin{tabular}{|c|c|c|}
\hline Champignons & $\begin{array}{l}\text { Fréquence } \\
\text { (p. 100) }\end{array}$ & $\begin{array}{l}\text { Abondance } \\
\text { (germes/g) }\end{array}$ \\
\hline $\begin{array}{l}\text { Absidia corymbifera } \\
\text { Mucor circinelloides } \\
\text { Mucor hiemalis } \\
\text { Mucor mucedo } \\
\text { Mucor pusillus } \\
\text { Mucor racemosus } \\
\text { Rhizopus nigricans } \\
\text { Thamnidium elegans } \\
\text { Neurospora sitophila } \\
\text { Aspergillus candidus } \\
\text { Aspergillus flavus } \\
\text { Aspergillus fumigatus } \\
\text { Aspergillus gr. glaucus } \\
\text { Aspergillus nidulans } \\
\text { Aspergillus niger } \\
\text { Aspergillus niveus } \\
\text { Aspergillus ochraceus } \\
\text { Aspergillus versicolor } \\
\text { Aspergillus wentii } \\
\text { Penicillium caseicolum } \\
\text { Penicillium chrysogenum } \\
\text { Penicillium citrinum } \\
\text { Penicillium cyclopium } \\
\text { Penicillium expansum } \\
\text { Penicillium oxalicum } \\
\text { Penicilium spinulosum } \\
\text { Penicillium stoloniferum } \\
\text { Penicillium viridicatum } \\
\text { Penicillium sp. } \\
\text { Paecilomyces variotii } \\
\text { Trichoderma viride } \\
\text { Chrysosporium pannorum } \\
\text { Scopulariopsis brevicaulis } \\
\text { Scopulariopsis candida } \\
\text { Wallemia sebi } \\
\text { Pullularia pullulans } \\
\text { Cladosporium cladosporioides } \\
\text { Alternaria consortiale } \\
\text { Phoma sp. } \\
\text { Geotrichum candidum } \\
\text { Mycélium stérile }\end{array}$ & $\begin{array}{c}13 \\
6 \\
\text { traces } \\
3 \\
3 \\
6 \\
10 \\
\text { traces } \\
3 \\
6 \\
23 \\
13 \\
50 \\
6 \\
16 \\
\text { traces } \\
10 \\
20 \\
10 \\
\text { traces } \\
10 \\
3 \\
16 \\
3 \\
10 \\
10 \\
33 \\
26 \\
20 \\
10 \\
3 \\
\text { traces } \\
13 \\
10 \\
6 \\
3 \\
26 \\
3 \\
\text { traces } \\
3 \\
3 \\
\end{array}$ & $\begin{array}{r}202 \\
119 \\
\\
200 \\
60 \\
81 \\
130 \\
\\
200 \\
83 \\
102 \\
28 \\
451 \\
30 \\
630 \\
\\
26 \\
2210 \\
71 \\
\\
140 \\
10 \\
28880 \\
30 \\
986 \\
1250 \\
5242 \\
4831 \\
35 \\
150 \\
125 \\
31 \\
33 \\
33 \\
100 \\
10 \\
350 \\
30 \\
10 \\
10\end{array}$ \\
\hline
\end{tabular}


On constate que 41 espèces de moisissures y ont été détectées. Les plus fréquentes sont :

Aspergillus du groupe glaucus (50 p. 100 des cas), puis

Penicillium stoloniferum (33 p. 100),

Penicillium viridicatum (26 p. 100),

Cladosporium cladosporioides (26 p. 100).

L'Aspergillus flavus ne vient qu'en $5^{\mathrm{e}}$ position avec 23 p. 100 des cas examinés.

Quand elles sont présentes, celles qui sporulent le plus abondamment (et qui constituent donc une menace d'envahissement facile du milieu) sont :

Penicillium cyclopium avec en moyenne 28880 germes par gramme d'échantillon (maximum : 96000 germes/g),

Penicillium stoloniferum avec 5242 germes/g,

Penicillium viridicatum avec 4831 germes/g,

Aspergillus versicolor avec 210 germes/g,

L'Aspergillus flavus vient loin derrière avec 102 germes/g.

Sur le plan toxicologique :

- les Aspergillus du groupe glaucus sont réputés causer des entérites et des baisses de rendement ; on en a notamment constaté chez les animaux de basse-cour ;

- le Penicillium stoloniferum est rendu responsable, par l'acide mycophénolique qu'il produit, d'une action toxique sur les leucocytes et de troubles d'anémie ;

- le Penicillium viridicatum provoque des accidents de néphrotoxicoses qui ont notamment été constatés au Danemark depuis 1928 ; le principe toxique qu'il élabore serait essentiellement de la citrinine, accessoirement de l'acide oxalique et une substance hépatotoxique encore mal déterminée (Krogh et Hasselager, 1968, 1970 ; Friss, Hasselager et Krogh, 1969) ;

- le Penicillium cyclopium élabore de nombreux composés toxiques, notamment des substances trémorgènes, neurotoxines provoquant des convulsions (Wilson et al., 1968 ; Hou et al., 1971) et de l'acide pénicillique quı agit sur le cœur à la manière de la digitaline;

- l'Aspergillus versicolor produit surtout de la stérigmatocystine, un composé carcinogène proche des aflatoxines.

Effectivement, certains de ces échantillons nous avaient été transmis par des vétérinaires qui avaient notamment constaté :

- de fortes diarrhées, des symptômes digestifs divers chez des veaux pour les échantillons où proliféraient les Aspergillus du groupe glaucus ;

- des symptômes nerveux pour des poudres lactées riches en Penicillium cyclopium, etc. 
La corrélation paraît évidente et une forte suspicion pèse sur ces poudres de lait qui étaient destinées à l'alimentation animale. Cependant, la recherche des mycotoxines n'ayant pas été effectuée, on ne peut apporter qu'une présomption de toxicité pour certains échantillons.

Ce sont sensiblement les mêmes espèces de moisissures qui avaient été mises en évidence dans les laits en poudre en Angleterre par Cooke et Brazis (1968).

\section{Lait concentré}

Si des précautions d'hygiène ne sont pas prises lors du scellement des boîtes de lait concentré, on peut avoir un développement d'Aspergillus repens, une espèce du groupe glaucus qui affectionne les milieux riches en sucres (Rogers et al., 1920).

\section{Fromages}

Il y a de nombreux types de fromages et la préparation de beaucoup d'entre eux fait appel à des champignons.

La pollution ambiante des industries fromagères est elle-même à l'origine de nombreuses contaminations par des moisissures indésirables ; la plupart ne causent que des défauts d'aspect, certaines nuisent aux qualités nutritionnelles ou organoleptiques des fromages (Moreau M., 1976).

La présence de moisissures est cependant nécessaire à de nombreux fromages.

Le plus célèbre d'entre eux, le Camembert, est préparé à l'aide du Penicillium caseicolum. Depuis que l'on consomme ce fromage, aucun cas d'intoxication ne semble avoir été signalé. Or, Gibel, Wegner et Wildner ont obtenu en 1971 la production de cancer chez des rats Wistar par application orale ou sous-cutanée d'extraits de culture d'un Penicillium qu'ils nomment curieusement Penicillium camemberti var. candidum. Le Penicillium camemberti Thom $(=P e-$ nicillium album Preuss) était l'espèce qui, antérieurement au Penicillium caseicolum était utilisée par les fromagers dans la préparation des Camemberts ; ils l'ont abandonné car $12 \mathrm{j}$ après son ensemencement sur les fromages il prenait une coloration légèrement bleutée qui déplaisait aux consommateurs. Par ailleurs, les fromagers appellent Penicillium candidum Link le Penicillium que les mycologues nomment $P$. caseicolum Bainier, Quel est exactement le champignon étudié par les auteurs allemands ? S'agit-il d'une souche particulière, d'un mutant à pouvoir carcinogène ? Nous l'ignorons, mais il serait bien utile d'y voir plus clair dans ce domaine. 
Il serait peut-être souhaitable d'envisager, à l'image de ce qui commence à se pratiquer en Allemagne, la mise en service dans le commerce de souches sélectionnées de Penicillium caseicolum, remplissant évidemment les meilleures conditions pour la préparation des fromages et qui auraient été testées pour certifier l'absence de pouvoir toxinogène. Mais il convient de savoir que l'étude d'une toxicité chronique exige des essais nombreux et fort longs.

Dans la préparation du fromage de Saint-Nectaire interviennent de nombreux champignons, récemment étudiés par Dale et Guillot (1971). Une étude plus précise des souches utilisées pourrait permettre de reconnaître si elles sont ou non toxinogènes. Cependant la préparation de ce fromage est actuellement surtout artisanale, d'où résulte une difficulté à réunir une collection convenable de souches.

Parmi les accidents de fabrication signalés figurent :

- le Penicillium cyclopium connu pour ses actions trémorgéniques ;

- le Penicillium brevicompactum dangereux par l'acide mycophénolique ;

- l'Aspergillus flavus susceptible d'élaborer des aflatoxines.

Près de 350 espèces de moisissures ont été reconnues par Bullermann et Olivigni (1974) sur le fromage de Cheddar : 82 p. 100 appartiennent au genre Penicillium, 6,6 p. 100 au genre Aspergillus. Beaucoup d'entre elles ont été reconnues toxinogènes par ces auteurs lorsque des extraits de leurs cultures sont injectés à des embryons de poulets. Mais la preuve n'a pas été apportée que les toxines se forment quand ces champignons se développent sur le fromage.

Dans la préparation des fromages tels que le Roquefort, le bleu d'Auvergne, le bleu des Causses, la fourme d'Ambert, le Gorgonzola, etc. intervient le Penicillium roqueforti Thom.

Au Japon, en 1956, on a attribué au Penicillium roqueforti la mort brutale de bétail (Tsubaki, 1956). Ce champignon s'était développé dans des grains ensilés.

Certaines souches, notamment celle nommée MR 212-2 isolée de farine de riz, ont particulièrement retenu l'attention, Kanota (1968, 1970) en a extrait trois toxines :

- une toxine 1, de formule $\mathrm{C}_{7} \mathrm{H}_{11} \mathrm{O}_{7}$ ou $\mathrm{C}_{7} \mathrm{H}_{12} \mathrm{O}_{7}$ possédant une fonction lactone, de toxicité relativement faible ;

- une toxine 2, capable de provoquer de graves altérations du foie et des hémorragies dans le tractus digestif ;

- une toxine 3, de toxicité assez faible. 
Wei et al. (1973) ont isolé une mycotoxine du Penicillium roqueforti : la PR toxine. Sa formule a été ensuite définie (Wei et al., 1975) et confirmée (Moreau S., Frayssinet et Jemmali, 1975) ; il s'agit d'un sesquiterpène $\mathrm{C}_{2 \pi} \mathrm{H}_{2} \mathrm{O}_{6}$.

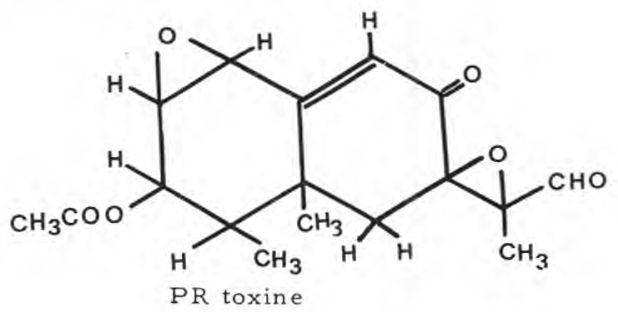

La PR toxine n'a jamais été mise en évidence dans le fromage de Roquefort. Des recherches menées tant en France (Crouzet, inédit) qu'en Allemagne (Frank, 1974) l'ont prouvé. D'après des essais actuellement en cours, certaines souches de Penicillium roqueforti ne forment cette toxine que dans des conditions très précises (milieu de culture enrichi à 15 p. 100 de saccharose, cultures en milieu aéré, $\mathrm{pH}$ constant proche de 4,5 , etc.) fort différentes de celles qui sont requises pour la préparation du fromage.

D'autres métabolites toxiques ont été récemment isolés des cultures de Penicillium roqueforti par Scott (publication en cours) d'une part, par Ohmomo et al. (1975) d'autre part. Ces derniers ont mis en évidence de la festuclavine, proche de l'alcaloïde de l'ergot de seigle, et trois autres alcaloïdes à noyau indole : les roquefortines A, B, C.

Selon les auteurs japonais, ces toxines pourraient être présentes dans divers fromages de type Roquefort, mais à des doses " insignifiantes » $(0,2$ à $3,6 \mathrm{mg}$ de roquefortine $\mathrm{A}$ par $\mathrm{kg}$ de fromage).

Les études se poursuivent en ce domaine.

En Espagne, on a constaté le développement d'Aspergillus flavus et d'Aspergillus parasiticus sur du fromage "Manchego ", ainsi que sur le chorizo et les olives noires et on a détecté des aflatoxines qu'ils produisaient (Sanz Perez et al., 1973).

Il ne faut pas négliger l'apport possible de mycotoxines par des épices et fines herbes qui sont introduites depuis peu dans divers fromages et qui, par leur pollution souvent importante par moisissures, provoquent de nombreux accidents de fabrication (Jacquet et Teherani, 1974).

Ainsi a-t-on reconnu, chez les fromages, tant comme utiles à leur préparation que responsables d'accidents de fabrication, des moisis- 
sures très variées (Le Gall, 1974) parmi lesquelles certaines souches ont, hors des fromages, été reconnues toxinogènes.

\section{Beurre}

Lors de la préparation du beurre, diverses moisissures peuvent jouer un rôle néfaste ; on en a dénombré plus d'une cinquantaine (Sasaki, 1950). L'Aspergillus repens agit surtout comme lipolytique, les Cladosporium herbarum et Oospora suaveolens sont particulièrement fréquents; l'Alternaria tenuis et des Fusarium sont abondants en été ; l'Aspergillus flavus est assez commun dans certaines régions.

Aucune étude toxicologique n'a été réalisée. Des précautions d'hygiène convenables dans les locaux de fabrication suffisent généralement à éviter les contaminations par ces moisissures.

\section{CONCLUSIONS}

Nos connaissances sur les mycotoxines sont encore toutes récentes. Leur développement est lié à l'évolution rapide des techniques d'élevage industriel.

Peu à peu on s'est avisé que si ces toxines avaient un effet (surtout intoxications aiguës, plus rarement chroniques) sur les animaux, elles risquaient de jouer un rôle en alimentation humaine (surtout intoxications chroniques en raison de la diversité de nos aliments).

Parmi les denrées alimentaires susceptibles de nous apporter des mycotoxines figurent les produits laitiers. C'est là un domaine d'autant plus important que le lait est une des bases de l'alimentation infantile.

Sans nier le risque encouru, il ne faut sans doute pas exagérer le danger. Certains ne manqueront pas d'utiliser les données scientifiques à des fins mercantiles. Mais là, comme ailleurs, le bon sens et un juste équilibre doivent prévaloir.

Il convient de rester attentifs devant de tels problèmes et il est nécessaire de poursuivre des recherches dans ce sujet d'actualité de l'hygiène alimentaire.

\section{R és u mé}

Les vaches laitières sont peu sensibles aux effets de l'aflatoxine $B_{1}$ mais une partie de cette mycotoxine qu'elles ingèrent avec leurs aliments est métabolisée dans le lait sous forme d'aflatoxine $\mathbf{M}_{1}$, 
hépatotoxique et cancérogène, ce qui peut être dangereux pour les consommateurs, surtout les jeunes enfants.

Sur les poudres de lait se développent de nombreuses moisissures dont beaucoup sont réputées toxinogènes.

Certaines souches de Penicillium du Camembert pourraient, selon certains auteurs, être toxiques. Plusieurs mycotoxines ont été reconnues parmi les produits élaborés par le Penicillium roqueforti; la principale d'entre elles, nommée PR toxine, ne peut être produite dans les conditions normales de fabrication du fromage de Roquefort.

Sans nier le risque encouru en relation avec les mycotoxines dans les produits laitiers, il ne faut pas en exagérer le danger.

\section{S u m m a r y}

\section{MYCOTOXINS IN DAIRY PRODUCTS}

Lactating cows are not very affected by aflatoxin $B_{1}$. Nethertheless a part of that mycotoxin, when contained in cow food, is metabolized and found in milk as aflatoxin $\mathbf{M}_{1}$, which is hepatotoxic and carcinogenic and could be dangerous for human health, specially for young children.

Many molds are developed in milk powders. Some of them are presumed to be toxinogenic.

According to some authors some strains of Penicillium of Camembert cheese could be toxic. Many mycotoxins have been identified in the elaborated products of Penicillium roqueforti, but the major, known as PR Toxin, could not be produced in the normal conditions of Roquefort cheese making.

The danger of mycotoxins in dairy products should be carefully considered but not overmagnified.

Reçu pour publication en février 1976.

\section{Bibliographie}

Abrams (L.) (1965). - Mycotoxicoses. J. S. Afr. Veter. Med. Ass., t. XXXVI, 5-13.

Allcroft (R.) and CARnaghan (R. B. A.) (1962). - Groundnut toxicity. Aspergillus flavus toxin (aflatoxin) in animal products : preliminary communication. Vet. Rec., t. LXXIV, 863-864.

Allcroft (R.) and Carnaghan (R. B. A.) (1963.. - Groundnut toxicity ; an examination for toxin in human food products from animals feed toxic groundnut meal. Vet. Rec., t. LXXV, 259-263.

Allcroft (R.) and CARNaghan (R. B. A.) (1963). - Toxic products in groundnuts : biological effects. Chemistry and Industry, t. II, 50-53. 
Allcroft (R.) and Lewis (G.) (1963). - Groundnut (meal) toxicity in cattle : experimental poisening of calves and a report on clinical effects in older cattle. Vet. Rec., t. LXXV, 487-494.

Allcroft (R.) and RoBerts (B. A.) (1968). - Toxic groundnut meal; the relationships between $B_{1}$ intake by cows and excretion of aflatoxin $\mathbf{M}_{1}$ in milk. Vet. Rec., t. LXXXII, 116-118.

Alicroft (R.), RoBERTS (B. A.) and LlyoYd (M. K.) (1968). - Excretion of aflatoxin in a lactating cow. Food Cosmet. Toxicol., t. VI, 619-625.

Allcroft (R.), Rogers (H.), Lewis (G.), Nabney (J.) and Best (P. E.) (1966). Metabolism of aflatoxin in sheep : excretion of the " milk toxin ". Nature, G.B., t. CCIX, 154-155.

BASSIR (O.) and OSIYEMI (F.) (1967). - Biliary excretion of aflatoxin in the rat after a single dose (Aspergillus flavus). Nature, G.B., t. CCXV, 882.

Büchi (G.) and WeinreB (S. M.) (1969). The total synthesis of racemic aflatoxin $\mathrm{M}_{1}$ (milk toxin).J. Am. Chem. Soc., t. XCI, 5408-5409.

Bullermann (L. B.) and Olivigni (F.J.) (1964). - Mycotoxin producing-potential of molds isolated from cheddar cheese. J. Food. Sci., t: XXXIX, 1166-1168.

ButLeR (W. H.) and CLIFFoRd (J. L.) (1965). - Extraction of aflatoxin from rat liver. Nature, t. CCVI, 1045-1046.

Campbell (T. C.) and Salamat (L.) (1971). - Aflatoxin ingestion and excretion by humans. In PuRchase (I. F. K.). Mycotoxins in human health, 271-280.

Chou Ming-Wu and Tung Ta-Chang (1969). - Aflatoxin $B_{1}$ in the excretion of aflatoxin poisoned rats. I. Formosan Med. Ass., t. LXVIII, 389-391.

Clegg (F. G.) and BRYson (H.) (1962). - An outbreak of poisoning in store cattle attributed to Brazilian groundnut meal. Vet. Rec., t. LXXIV, 992-994.

COOKE (W. B.) and BRAZIS (A.R.) (1968). - Occurrence of molds and yeasts in dairy products. Mycopathol. Mycol. Appl., t. XXXV, 281-289.

Dale (G.) et Guillot (J.) (1971). - Contribution à l'étude de la mycoflore du fromage de Saint-Nectaire et de la physiologie du Geotrichum candidum Link. C.R. Séances Soc. Biol., t. CLXV, 309-316.

De Iong (H.), Van Pelt (J. G.), Ord (W. O.) and Barrett (C. B.) (1964). - A semi quantitative determination of aflatoxin $B_{1}$ in groundnut meal, groundnuts and peanut butter. Vet. Rec., t. LXXVI, 901-903.

De Iongh (H.), Vles (R. Q.), Barrett (C.) and Van Pelt (J. G.) (1965). - Milk of mammals fed an aflatoxin-containing diet. Nature, t. CCII, 466-467.

De Iongh (H.), Vles (R. Q.) and De Vogel (P.) (1965). - The occurrence and detection of aflatoxin in food. Mycotoxin in Foodstuffs, 235-245.

Delage (S.) et Fehr (P.) (1967). - Contribution à l'étude des répercussions de la présence d'aflatoxine dans les régimes d'animaux domestiques. Ind. Alim. Anim., $\mathrm{n}^{\circ} 183,44-47$.

Fehr (P. M.), Delage (J.) et Richir (C.) (1974). - Répercussions de l'ingestion d'aflatoxine sur le lapin en croissance. Cah. Nutr. Diét., t. V, 62-64.

FrANK (H. K.) (1974). - Bundesforschungsanstalt für Ernährung in Karlsruhe. Jahresbericht, $92 \mathrm{p}$.

FrANK (H. K.) (1974). - Aflatoxine. Bildungsbedingungen Eigenschaften und Bedeutung für die Lebenswirtschaft. Schriftenreihe des Bundes für Lebensmittelrecht und Lebensmittelkunde, $\mathrm{n}^{\circ} 76,126 \mathrm{p}$.

Fris (P.), Hasselager (E.) and KroGh (P.) (1969). - Isolation of citrinin and oxalic acid from Penicillium viridicatum Westling and their nephrotoxicity in rats and pigs. Acta Path. Microbiol. Scand., t. LXVII, 559-560.

Gibel (W.), Wegner (K.) und Wildner (G. P.) (1971). - Experimentelle Untersuchungen zur Frage einer kanzerogenen Wirkung von Penicillium camemberti var. candidum. Archiv. f. Geschwulstforsch., t. XXXVIII, 1-6. 
Holzapfel (C. W.), Steyn (P. S.) and Purchase (I. F. H.) (1966). - Isolation and structure of aflatoxins $\mathrm{M}_{1}$ and $\mathrm{M}_{2}$. Tetrahedron Letters, t. XXV, 2799-2803.

Hou (C. T.), Ciegler (A.) and Hesseltine (C. W.) (1971). - Tremorgenic toxins from Penicillia. II. A new tremorgenic toxin, tremortin B, from Penicillium palitans. Can. J. Microbiol., t. XVII, 599-601.

Jacouet (J.), Boutibonnes (P.), Teherani (A.) et Tantoui(A.), (1971). - Sur la fréquence des moisissures du genre Aspergillus. Aperçu sur la présence des flavacoumarines (aflatoxines) dans les aliments. Bull. Acad. Nat. Méd., t. CLV, 268-273.

Jacquet (J.) et Teherani (M.) (1974). - Présence exceptionnelle de l'aflatoxine dans certains produits d'origine animale. Rôle possible du poivre. Bull. Acad. Vétér. Fr., t. XLVII, 313-315.

JoNES (F. T.) and LEE (K. S.) (1968), - Note on aflatoxin $\mathrm{M}_{1}$ : optical and X-ray crystallographic data. J. Ass. Off. Anal. Chem., t. LI, 610.

Jung (M.) und HanSSEN (E.) (1974). - Ueber das Vorkommen von Aflatoxin M in Trockenmilcherzeugnissen. Food Cosmet. Toxicol., t. XII, 131-138.

Kanota (K) (1968). - Studies on toxic metabolites of Penicillium roqueforti. Proc. 1st. U.S.-Jap. Conf. on Toxic microorganisms, Honolulu, 129.

Kanota (K.) (1970). - Studies on toxic metabolites of Penicillium roqueforti. In Herzberg (M.), Toxic Microorganisms, 129-132.

Kiermeier(F.) (197e), - Zum Nachweis von Aflatoxinen in Käse. Zeitschr.f. Lebensmitteluntersuch, u. Forsch., t. CXLIV, 293-297.

KIERMEIER (F.) (1971). - Zur Aflatoxin-Bildung auf Käse. Ernährungsforschung, t. XVI, 519-526.

KIERMEIER (F.) (1971). - Zur Aflatoxin-Bildung in Milch und Milchprodukten. IV. Modellversuche mit Milchpulver. Zeitschr. f. Lebensmitteluntersuch. $\mu$. Forsch., t. CXLVI, 262-265.

KieRMEIER (F.) (1972). - Schwierigkeiten bei der quantitativen Bestimmung der Aflatoxine. Zeitschr. f. Lebensmitteluntersuch. u. Forsch., t. CXLVIII, 331-341.

KIERMEIER (F.). - Aflatoxin residues in fluid milk. IUPAC Symposium ; Control of Mycotoxins, Kungälv (Göteborg), Sweden, 12, 21-22 août 1972.

Kiermeier (F.). - Mykotoxine in Milch und Milchprodukten. Mycotoxin Symposium, Karlsruhe, 3 p., 28 sept 1972 und Zeitschr. f. Lebensmitteluntersuch. $u$. Forsch., t. CLI, 237-240, 1973.

Kiermeier (F.) (1973). - Ueber die Aflatoxin-M-Ausscheidung in der Kuhmilch in

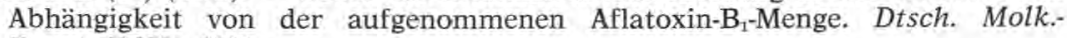
Ztg., t. XCIV, 1098.

Kiermeier (F.) und Behringer (G.) (1972). - Zur Aflatoxinbildung in Milch und Milchprodukten. VII. Zur Problematik der Probenahme in aflatoxinhaltigen Lebensmitteln. Zeitschr. f. Lebensmittelunters, u. Forsch., t. CXLVIII, 72-76.

Krermeier (F.) und Behringer (G.) (1973). - Einfluss des pH Wertes auf die Aflatoxinbildung in Modell-versuchen mit Milchpulver. Zeitschr. f. Lebensmittelunters. u. Forsch., t CLI, 392-397.

Kiermeier (F.) und Bohm (S.) (1971). - Zur Aflatoxinbildung in Milch und Milchprodukten. V. Anwendung des Hühnerembryo-Testes zur Sicherung des dünnschichtchromatographischen Aflatoxin-Nachweises in Käsen. Zeitschr. f. Lebensmittelunters. $u$. Forsch., $t$ CXLVII, 61-64.

KieRMeier (F.) und Groll (D.) (1970). - Zur Bestimmung von Aflatoxin B $B_{1}$ in Käse. Zeitschr. f. Lebensmittelunters, u. Forsch., t. CXLII, 120-123.

Kiermeier (F.) und Groll (D.) (1970). - Zur Aflatoxin B-Bildung in Käsen. Zeitschr. f. Lebensmittelunters. u. Forsch., t. CXLIII, 81-89.

KIERMEIER (F.) und MücKE (W.) (1971). - Sprühreagens zum Nachweis von Aflatoxinen. Zeitschr. f. Lebensmittelunters. u. Forsch., t. CXLVI, 329-331. 
Kiermeier (F.), REINhardt (V.) und Behringer (G.) (1975). - Zum Vorkommen von Aflatoxinen in rohmilch. Dtsch. Lebensmitt.-Rundsch., t. LXXI, 35-38.

KIERMEIER (F.) und RUMPF (S.) (1975). - Ueber das Schicksal des Aflatoxins bei der Schmelzkäseherstellung. Zeit. $f$. Lebensmittelunters. $u$. Forsch., t CLVII, 211-216.

KIERMEIER (F.) und Zierer (E.) (1975). - Zur Wirkung von Pimaricin auf Schimmelpilze und deren Aflatoxinbildung bei Käsen. Zeitschr. f. Lebensmittelunters. $u$. Forsch., t. CLVII, 253-262.

Krogh (P.) and Hasselager (E.) (1968). - Studies on fungal nephrotoxicity. Kong. Vet. Landbohojsk. Arsskr. 198-214.

Krogh (P.) and Hasselager (E.) (1970). - Svampebetingede sygdomme hos husdyr, med saerlig henblick pa mykotoksikoser hos danske svin. Nord. Vet.-Med., t. XXII, 141-160.

Lafont (P.) et Lafont (J.) (1975). - Elimination d'aflatoxine par la mamelle chez la vache. Cahiers Nutr. Diét., t. X, 55-57.

LE Gall (D.) (1974). - Les moisissures utiles et nuisibles dans l'industrie fromagère. Travail de recherches bibliographiques. Maîtrise de Sciences et Techniques «Biologie appliquée aux Industries Agro-alimentaires». Brest.

Lewis (G.), Markson (L. M.) and Allcroft (R.) (1967). - The effect of feeding toxic groundnut meal to sheep over a period of five years. Vet. Rec., t. LXXX, 312-314.

Masri (M. S.), Garcia (V. C.) and PaGe (J.R.) (1969). - The aflatoxin M content of milk from cows fed known amounts of aflatoxin. Vet. Rec., t. LXXXIV, 146-147.

Masri (M. S.), Lundin (R. E.), Page (J.R.) and Garcia (V. G.) (1967). - Crystalline aflatoxin $\mathrm{M}_{1}$ from urine and milk. Nature, G.B., t. CCXV, 753-755.

MoREAU (C.) (1974). - Moisissures toxiques dans l'alimentation, $2^{e}$ éd., 471 p., Masson éd., Paris.

Moreau (M.) (1976). - Pollution ambiante et accidents de fabrication par moisissures des fromages à pâte molle. Ind. alim. agric. (sous presse).

Moreau (S.), Frayssinet (C.) et Jemmali (M.) (1975). - La P.R. toxine. Colloque INSERM " Les Mycotoxines ", t. XLVI, 89-92.

Nabney (J.), Burbage (M. B.), Allcroft (R.) and Lewis (C.) (1966). - Metabolism of aflatoxin in sheep ; excretion pattern in the lacting ewe. Indian J. Chem., t. IV, 11-17.

NabNEY (J.) and NesbitT (B. F.) (1964). - Determination of the aflatoxins. Nature, G.B., t. CCIII, 862 .

Neumann-Kleinpaul (A.) und Terplan (G.) (1972). - Zum Vorkommen von Aflatoxin $\mathrm{M}_{1}$ in Trockenmilchprodukten. Arch. Lebensmittelhyg., t. XXIII, 128-132.

Ohmomo (S.), Sato (T.), Utagawa (T.) and ABe (M.) (1975). - Isolation of festuclavine and three new indole alkaloids, roquefortine A, B and $\mathrm{C}$ from cultures of Penicillium roqueforti. Agr. Biol. Chem. t. XXXIX, 1333-1334.

Pai (M. R.), BaI (N. J.) and Vankitasubramanian (T. A.) (1975). - Production of aflatoxin $\mathrm{M}$ in a liquid medium. Appl. Microbiol., t. XXIX, 850-851.

Patterson (D. S. P). and Allcroft (R.) (1970). - Metabolism of aflatoxin in susceptible and resistant animal species. Food Cosmet. Toxicol., t. VIII, 43-53.

Polan (C. E.), Hayes (J. R.) and Campbell (T. C.) (1974), - Consumption and fate of aflatoxin $\mathrm{B}_{1}$ by lactating cows. J. Agric. Food Chem., t. XXII, 635-638.

Purchase (I. F. H.) (1967). - Acute toxicity of aflatoxins $M_{1}$ and $M_{2}$ in one-dayold ducklings. Food Cosmet. Toxicol., t. V, 339-342.

PuRchase (I. F. H.) and VoRSTER (L. J.) (1968), - Aflatoxin carcino in commercial milk samples. S. Afr. Med. J., t. XLII, 219. 
Roberts (B. A.) and Allcroft (R.) (1968). - A note on the semi-quantitative estimation of aflatoxin $M_{1}$ in liquid milk by thin layer chromatography. Food Cosmet. Toxicol., t. VI, 339-340.

Rogers (A. E.), Dahlberg (A. O.) and Evans (A. E.) (1920). - The cause and control of "buttons " in sweet condensed milk. J. Dairy Sci., t. III, 122-129.

SASAKI (Y) (1950). - A study of molds in butter. J. Fac. Agric. Hokkaido Univ., t XLIX, 121-249.

Sanz Perez (B.), Palacios (A.), Sento (P.), Tormo Iguacel (J.) et Lopez Lorenzo (P.) (1937). - Investigacion de aflatoxinas en diversos alimentos espanoles. An. Bromatol., t. XXV, 297-319.

Sinnhuber (R. O.), Lee (D. J.), Wales (J. H.), Landers (M. K.) and Keyl (A. C.) (1970). - Aflatoxin $\mathbf{M}_{1}$ a potent liver carcinogen for rainbow trout. Fed. Proc., t. XXIX, 568.

Stubblefield (R. D.) and Shannon (G. M.) (1974). - Aflatoxin $\mathbf{M}_{1}$ : analysis in dairy products and distribution in dairy foods made from artificially contaminated milk. J. Ass. Off. Anal. Chem., t. LVIII, 847-851.

Stubblefield (R.) and Shannon (G.) (1974). - Collaborative study of methods for determination and chemical confirmation of aflatoxin $\mathbf{M}_{1}$ in dairy products. J. Ass. Off. Anal. Chem., t. LVII, 852-857.

Tsubaki (K.) (1976) - Penicillium isolated from toxic ensilage. Trans. Mycol. Soc. Japan, t. I, 6-7.

Wei (R. D.), Schnoes (H. K.), Hart (P. A.) and Strong (F. M.) (1975). - The structure of PR toxin, a mycotoxin from Penicillium roqueforti. Tetrahedron, t. XXXI, 109-114.

Wei (R. D.), Still (P.), Smalley (E. B.), Schnoes (H. K.) and Strong (F. M.). (1973). - Isolation and partial characterization of a mycotoxin from Penicillium roqueforti. Appl. Microbiol., t. XXV, 111-114.

Wilson (B. J.), Wilson (C. M.) and Hayes (A. W.) (1968). - Tremorgenic toxin from Penicillium cyclopium grown on food materials. Nature, G.B., t. CCXX, 77-78. 